\title{
Plebanski sectors of the Lorentzian 4-simplex amplitude
}

\section{Antonia Zipfel*}

Wydzial Fizyki Uniwersytetu Warszawskiego

E-mail: antonia.zipfel@fuw.edu.pl

The spin foam model is based on a BF-type action restricted by the simplicity constraint. However, the solutions to the simplicity constraint fall into five different sectors. In [1,2] it was argued that a certain mixing of these sectors and the freedom of choosing a tetrad orientation generates undesired terms in the asymptotic of the Euclidean EPRL-model and can be cured by an additional constraint. We here show that this is also the case for the Lorentzian model. This is joint work with J. Engle.

\section{References}

[1] J. Engle, A spin-foam vertex amplitude with the correct semiclassical limit, Phys.Lett. B724 (2013) 333-337.

[2] J. Engle, A proposed proper EPRL vertex amplitude, Phys.Rev D87 (2012) 084048.

Frontiers of Fundamental Physics 14 - FFP14,

15-18 July 2014

Aix Marseille University (AMU) Saint-Charles Campus, Marseille

\footnotetext{
*Speaker.
} 